\title{
GIST AND EGIST: REVIEW OF TWO RARE CASES
}

\author{
B. B. Gupta ${ }^{1}$, S. S. Changole ${ }^{2}$, V. Nanadagawali ${ }^{3}$, Chandratej Kadam ${ }^{4}$
}

\section{HOW TO CITE THIS ARTICLE:}

B. B. Gupta, S. S. Changole, V. Nanadagawali, Chandratej Kadam. "Gist and Egist: Review of two Rare Cases". Journal of Evolution of Medical and Dental Sciences 2014; Vol. 3, Issue 70, December 15; Page: 15012-15016, DOI: $10.14260 /$ jemds/2014/4019

\begin{abstract}
Mesenchymal tumors originating from the wall of the gastrointestinal tract have contributed to fascinating literature worldwide regarding their controversial histogenesis. Most common mesenchymal tumor arising in the gastrointestinal tract is gastrointestinal stromal tumor (GIST).[1,2] Apart from gastrointestinal tract, GISTs that involve omentum, mesentery and retroperitoneum are commonly known as extra gastrointestinal stromal tumors (EGISTs) Though there are many studies regarding various aspects of GISTs, yet primary EGISTs literature are limited. EGISTs are often included in large studies of stromal tumors, in which they account for less than $10 \%$ of overall cases. In most cases a preoperative diagnosis is not possible, and the patient undergoes a surgical operation for the generic diagnosis of "abdominal mass". Here were report two rare presentations in the form of non exophytic intraluminal GIST and huge EGIST.
\end{abstract}

KEYWORDS: GIST, EGIST, Non exophytic intraluminal.

CASE 1: INTRALUMINAL GIST: We report a case of $48 \mathrm{yr}$ old female resident of Nagpur, Maharashtra came to our institute with history of intermittent episodes of hematemesis since 6 months. It was associated with epigastric pain. She also had generalized weakness and easy fatigability since 2moths.On general examination pallor was present and there was no other significant finding. On routine blood investigations her HB was $6.0 \mathrm{mg} \%$ and rest with in normal limits. USG abdomen was not significant. She underwent upper GI scopy in view of intermittent hematemesis which showed an intraluminal mass of $5^{*} 5 \mathrm{~cm}$ over the greater curvature of stomach from which biopsy was taken and sent for HPE. There was no any active source of bleeding from that intraluminal mass or any other lesion up till duodenum. Histopath report was suggestive of? Gastrointestinal stromal tumor for which patient was advised a surgery.

After correction of anemia patient was taken for exploratory laprotomy. Intra operatively as seen in Fig 1a there was no evidence of any exophytic mass involving stomach, on palpating there was intraluminal growth over greater curvature for which partial Gastrectomy was done preserving the pylorus. On cut section there was pedunculated complete intraluminal mass with no exophytic extension and gross appearance was distinctly different for usual GIST tumours as shown in Fig1b. Specimen was sent for histopathological examination which later confirmed it to be a GIST.

Post operatively patient had no episodes of haematosis. Her HB was $11 \mathrm{gm} \%$ after $1 \mathrm{month}$. She was started on Imatinib and is under 1 monthly follow up and there are no signs of recurrence after 1yr. 


\section{CASE REPORT}

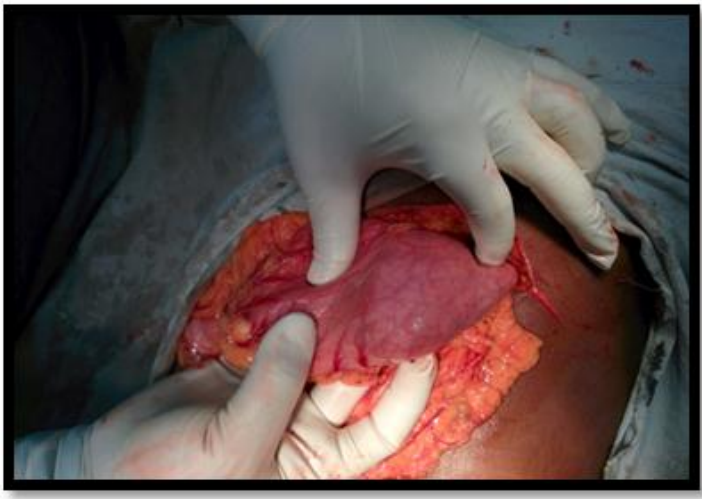

FIG. 1a: Showing non exophytic mass in stomach

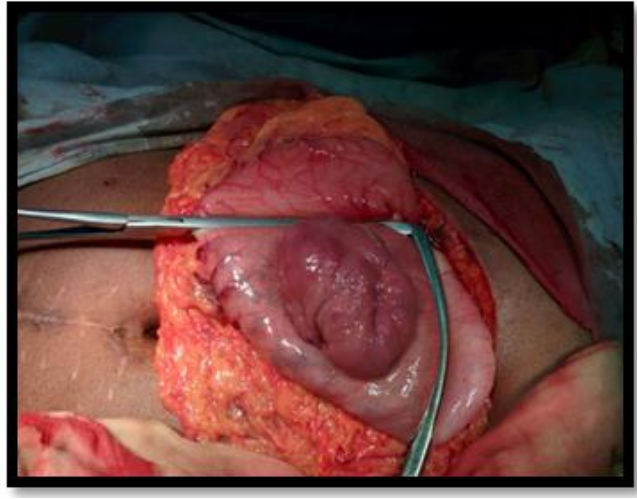

FIG. 1b: Showing intraluminal pedunculated mass

CASE 2 EXTRA GASTRO INTESTINAL STROMAL TUMOUR: We report second case of 55yrs female from Nanded Maharashtra came to our institute with chief complaints of lump in lower abdomen since three months associated with vague abdominal pain. She also had loss of weight and loss of appetite since 2 months. On examination there was huge lump of $18 \times 20 \mathrm{~cm}$ in umbilical region extending upto right lumbar and illiac region as shown in fig. 2.

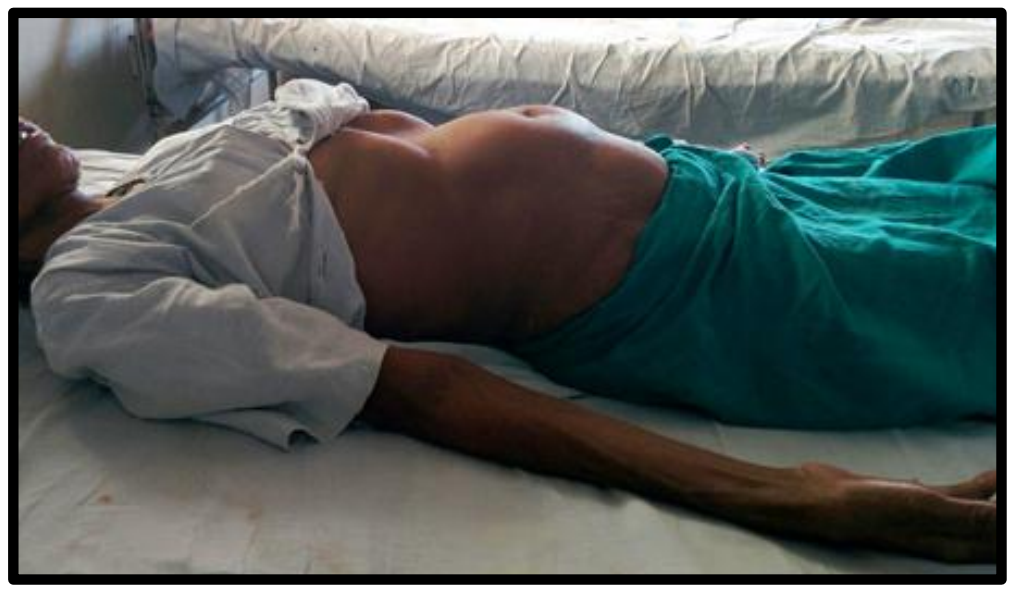

FIG. 2: Clinical photograph of patient

After routine blood investigations and X-ray chest which were with in normal limits, Radiological investigations were done which showed following results:

USG abdomen showed large lobulated heterogeneous mass lesion of size 15.9x11.9x15.2 in retoperitoneum mainly in Right lumbar umbilical and iliac region with minimal vascularity. There were multiple lymph nodes surrounding the lesion and multiple calcific foci with in the lesion, suggestive of neoplastic etiology? leiomyosarcoma? liposarcoma

CT scan as shown in fig3 was suggestive of large heterogeneously enhancing hypodense lesion in the mesentry mainly in right illiac fossa showing areas of necrosis and calcification within 


\section{CASE REPORT}

causing mass effect on the adjacent bowel loops with well-preserved fat planes s/o neoplastic etiology with multiple metastatic lymph nodes in mesentry.

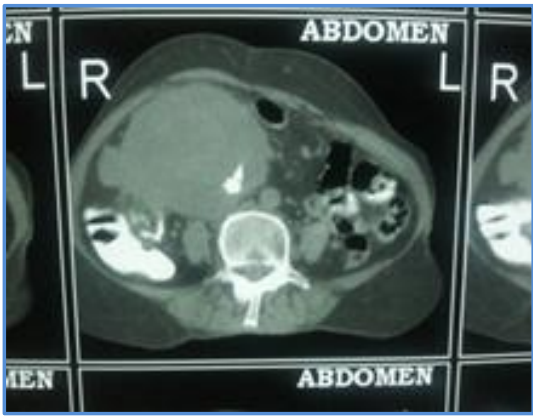

\section{FIG. 3: CT scan films showing large hypodense mass arising from mesentry}

USG guided FNAC from the lesion couldn't specify the tissue diagnosis as it was possibly? Spindle cell neoplasm?, low grade sarcoma?, malignant mesenchymal tumour. Depending upon the above findings decision to do exploratory laprotomy was taken. Intraoperatively as shown in the fig.4 there was huge mass of about $18 \times 20 \mathrm{~cm}$ attached to the base of mesentry without involving any bowel structures but displacing them. Mass was separated from surrounding structures and removed completely as shown in fig 5 and sent for histopathological examination.

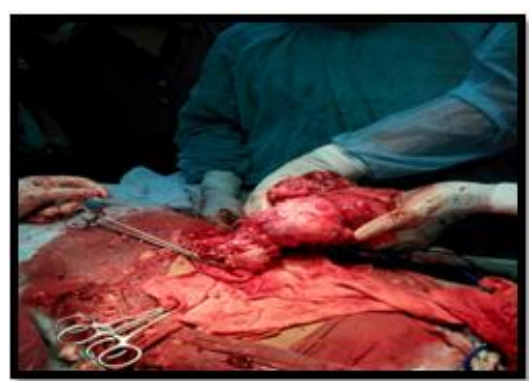

FIG. 4: Intraoperative photograph

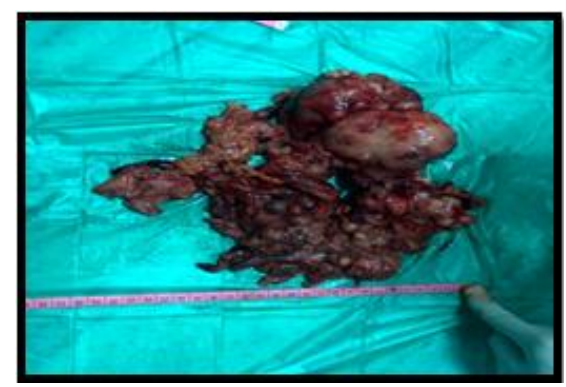

FIG. 5: Specimen photograph

Histopath report as shown in fig 6 was suggestive of mesenchymal tumour with spindle cell sacrcoma favouring MPNST (malignant peripheral nerve sheath tumour) and needed further immunohistochemistry for definitive typing.

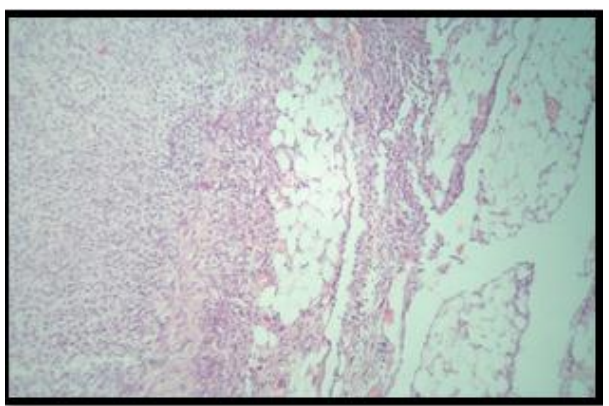

FIG. 6: Histopath suggestive of mesenchymal tumour with intermittent chondrotic deposition 
Immunohistochemistry studies showed exceptionally different results as shown in fig 7 and 8: tumor cells were strongly immunoactive for DOG1 and immunonegative for CD117 (c-kit)/CD34 and S100 Positive based on which it was classified as Gastrointestinal stromal tumor.

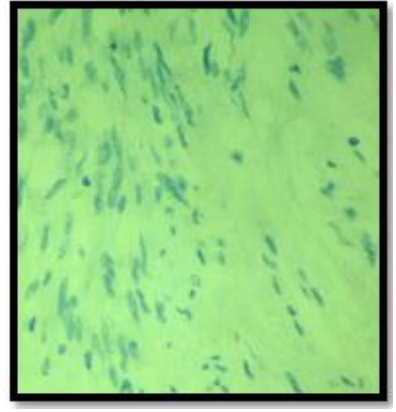

FIG. 7: Immunohistochemistry showing CD117 NEGATIVE

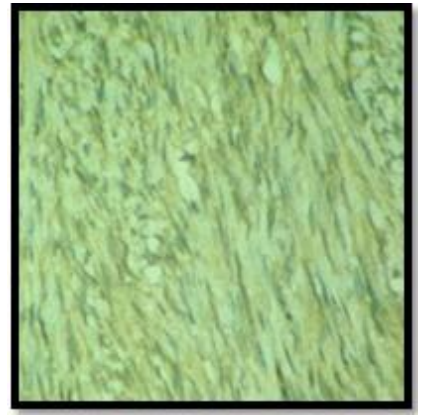

FIG. 8: Immunohistochemistry showing DOGI POSITIVE

Patient was given IMATINIB course and is under 2 mothly follow up and has not shown any signs of recurrence till now after 8months.

DISCUSSION: Classical gastrointestinal stromal tumor (GIST) is a non-epithelial neoplasm arising from the muscularis propria layer of the gastrointestinal tract leading to its tendency for exophytic growth. This is in contrast to other mesenchymal tumors like leiomyomas that involve the muscularis mucosae. GISTs most frequently occur in the stomach (70\%), followed by the small intestine (20-

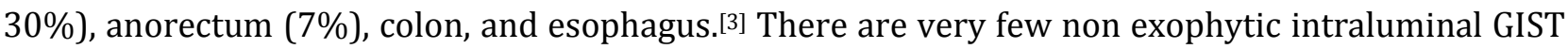
cases descirebed in literature. We present one such case which respond to complete resection followed by chemotherapy.

Extragastrointestinal stromal tumors (EGISTs) have no contact with the stomach and intestine, by definition, and typically occur in the omentum, mesentery or retroperitoneum. EGISTs have rarely been identified in the pancreas, diaphragm, spleen, pelvis or abdominal wall. Primary EGISTs are exceedingly rare. They usually present as enlarging masses of variable duration in adults. These tumors tend to be large, most measure more than $10 \mathrm{~cm}$ at the time of detection. ${ }^{[4]}$ On the basis of imaging findings alone it may be very difficult to distinguish GIST from other retroperitoneal mesenchymal neoplasms such as malignant fibrous histiocytoma, liposarcoma, leiomyosarcoma, and fibrosarcoma.[5] Gross appearance of EGISTs varies from firm, fleshy gray-red masses to cystic ones. They lack the whorled appearance of the conventional smooth muscle tumors as seen in fig 5 .

Microscopically EGISTs exhibit epithelioid, spindle cell pattern or combination of both patterns. Heterologous elements like cartilage and bone are very rare in EGIST as seen in fig 6.

Immunohistochemical characteristics of EGISTs are similar to GISTs, the majority of both having mutually exclusive gain-of-function KIT/ platelet-derived growth factor receptor-alpha (PDGFRA) mutations.[4,6] Another recent marker, DOG1 (discovered on GIST-1), is expressed in GISTs irrespective of KIT or PDGFRA mutation status.[7] In our case it was DOG1 positive but negative for CD117 as shown in fig 7,8. The literature regarding primary EGISTs are sparse, as majority are case reports. Reith et al. in one of the largest study described EGISTs in patients with a mean age of 58 years with female preponderance and majority being intraabdominal tumours presenting with abdominal pain which coincides with our case.[8] 
CONCLUSION: Non exophytic GIST and EGIST are rare tumours.EGISTs should be considered in the differential diagnosis of the mesenchymal tumors and immunohistochemistry helps to confirm the diagnosis. Considering the malignant potential all EGIST should be treated aggressively.

\section{REFERENCES:}

1. Miettinen M, Hala M, Sobin LH, Lasota J. Gastrointestinal stromal tumors of the jejunum and ileum. A clinicopathological, immunohistochemical and molecular genetics study of 906 cases before imatinib with long term follow up. Am J Surg Pathol 2006; 30: 477-89.

2. Miettinen M, Lasota J, Sobin LH. Gastrointestinal stromal tumors of the stomach in children and growing adults. A clinicopathological, immunohistochemical and molecular genetics study of 44 cases with long term follow up and review of literature. Am J Surg Pathol 2005; 29: 1373-81.

3. Casella C, Villanacci V, D’Adda F, Codazzi M, Salerni B. Primary extra-gastrointestinal stromal tumor of retroperitoneum. Clin Med Insights Oncol. 2012; 6: 189-97. [PMC free article] [PubMed]

4. Reith J, Goldblum JR, Weiss SW. Extragastrointestinal (soft tissue) stromal tumors: An analysis of 48 cases with emphasis on histologic predictors of outcome. Mod Pathol 2000; 13: 577-85.

5. 5. Gupta N, Mittal S, Lal N, Misra R, Kumar L, Bhalla S. A rare case of primary mesenteric gastrointestinal stromal tumor with metastasis to the cervix uteri. World J Surg Oncol 2007; 5: 137.

6. Van der Zwan SM, De Matteo RP. Gastrointestinal stromal tumor: 5 years later. Cancer 2005; 104: 1781-8.

7. West R, Corless C, Chen X, Rubin B, Subramanian S, Montgomery K, et al. The novel marker, DOG1, is expressed ubiquitously in gastrointestinal stromal tumors irrespective of KIT or PDGFRA mutation status. Am J Pathol 2004; 165: 107-13.

8. Miettinen M, Lasota J. Gastrointestinal stromal tumors (GISTs): Definition, occurrence, pathology, differential diagnosis and molecular genetics. Pol J Pathol 2003; 54: 3-24.

\section{AUTHORS:}

1. B. B. Gupta

2. S. S. Changole

3. V. Nanadagawali

4. Chandratej Kadam

\section{PARTICULARS OF CONTRIBUTORS:}

1. Professor, Department of General Surgery, Government Medical College, Nagpur.

2. Associate Professor, Department of General Surgery, Government Medical College, Nagpur.

3. Assistant Professor, Department of General Surgery, Government Medical College, Nagpur.
4. Junior Resident, Department of General Surgery, Government Medical College, Nagpur.

\section{NAME ADDRESS EMAIL ID OF THE CORRESPONDING AUTHOR:}

Dr. Chandratej Kadam, \# b-2, Anuradha Co. op. Housing Society Opp, Golf Club Rest House, Nashik, Maharashtra-422002, India.

Email: chandratejkadam@gmail.com

Date of Submission: 05/12/2014. Date of Peer Review: 06/12/2014. Date of Acceptance: 11/12/2014. Date of Publishing: 15/12/2014. 